del prodotto del concepimento per: 1) presenza dei geni letali e subletali; 2) incompatibilità sierologiche materno-fetali.

I fattori ambientali potranno essere rimossi con una accurata igiene della gravidanza, le cause ereditarie con opportune norme di eugenica, le malformazioni genitali, a seconda delle possibilità, con terapia chirurgica.

L'A. ha presentato, in appendice, dei dati statistici sulle malformazioni congenite forniti dall'Istituto Italiano di Statistica e dall'Alto Commissariato per la Sanità.

Gli Atti si aprono col testo, nell'originale francese e nella traduzione italiana, inglese, tedesca e spagnola dell'importante discorso rivolto ai congressisti da S.S. Pio XII di v.m., che affrontava le questioni morali connesse alla pratica della fecondazione artificiale e alla raccolta del seme ai fini delle ricerche di laboratorio.

\section{A. M.}

AKe Stenstedt: Involutional Malancholia An etiologic, clinical and social study of endogenous depression in later life, with special reference to genetic factors.

Acta Psych. et Neur. Scand. - Sup. 127, vol. 34, 1959.

Uno dei più gravi ostacoli delle ricerche psichiatriche $\dot{e}$ quello della impostazione scientifica del lavoro con la eliminazione degli elementi soggettivi involontari del ricercatore.

Ake Stenstedt ha cercato, attraverso il metodo genetico statistico di Larsson e Sjögren, di eliminare ogni possibile interferenza soggettiva costruendo una apparente fredda e schematica analisi di 307 casi di melanconia involutiva.

Tale sindrome viene negata da alcuni AA. specie tedeschi ed americani quale entità nosologica e ritenuta un episodio tardivo di depressione distimica. Tuttavia esistono alcuni caratteri, quale l'episodio unico insorgente a tarda età, la presenza di un delirio ipocondriaco o nichilistico, l'ansietà accompagnata da profon- da irrequietezza e agitazione, l'assenza di deterioramento a tipo senile che, per la loro costanza, posson circoscrivere la forma.

L'A. ha appunto selezionato i 307 casi con tali criteri suddividendo le varie espressioni della malattia, a seconda delle manifestazioni, in psicotiche, a tipo reazione disforica, a tipo reazione ipocondriaca e, nei 7 capitoli della lucida monografia, ne ha studiato i caratteri con particolare riguardo ai fattori genetici.

A tale scopo, oltre ai pazienti, esaminati in massima parte personalmente nei varii Ospedali, secondo uno schema che raggruppa alcune caratteristiche (età, sintomatologia, numero di attacchi, " elementi disturbanti », il quadro clinico, età di inizio ecc.) sono stati considerati il rischio per la malattia mentale e per altre anormalità tra $i$ genitori e collaterali a loro volta suddivisi in sottogruppi per i fattori etiologici rilevanti. Tali sottogruppi sono stati poi controllati e confrontati tra loro e in seguito con $\mathrm{i}$ dati della popolazione generale.

In una ma'attia che insorge in tarda età, quando gli agenti esogeni, ambientali, tossici, catabolici hanno avuto il tempo di esercitare una vasta azione morbigena è difficile poter sceverare e pesare l'importanza dei varii fattori alla ricerca di una etiologia unica e determinante.

I risultati ottenuti dall'analisi dei casi, nei diversi aspetti, ci rivelano $i$ seguenti dati interessanti :

Il $10 \%$ dei p. avevano un genitore con un disordine affettivo endogeno; $34 \%$ soffrivano di una grave forma psicotica mentre il resto di una più blanda depressione; $74 \%$ avevano avuto solo una crisi: $45 \%$ mostravano una deviazione della personalità; $25 \%$ erano divenuti depressi in seguito ad un trauma psichico e $36 \%$ in seguito ad altri fattori esogeni; $13 \%$ erano agitati; $20 \%$ decaduti e $10 \%$ con psicosi di negazione di Cotard; solo il $9 \%$ delle donne (237 dei pazienti) ebbero la loro crisi parallelemente alla menopausa. I celibi e $\mathrm{i}$ divorziati erano in maggior numero che non $i$ coniugati o vedovi; la classe sociale indifferente. 
La mortalità tra i p. fu superiore della media generale, come superiore il numero dei suicidi. A tal proposito è stato notato che tra $\mathrm{i}$ padri e i fratelli dei p. vi era un numero maggiore di suicidi che non nella media generale: Lo studio dei familiari dava la presenza di circa il doppio dei disordini affettivi allorchè paragonati con la popolazione generale $66 \%$ anzichè $3 \%$ ). Tuttavia una ereditarietà vera ha giocato solo in un numero esiguo un ruolo importante. I gemelli nel materiale esaminato sono 4 , tutti discordanti nella sintomatologia.

L'etiologia della forma, purtroppo, rimane ancora incerta e legata a fattori multipli sia endogeni che esogeni.

L'etiologia della forma, purtroppo, rimane ancora incerta e legata a fattori multipli sia endogeni che esogeni.

Nell'appendice, ciascun caso viene esaminato singolarmente nelle sue componenti sia sintomatologiche come genetiche.

I risultati non sono evidentemente determinanti e questo spiega l'incertezza dei varii $A A$. sia nella diagnosi come nella etiologia della sindrome involutiva. Anche se permane al !ettore, alla fine, intatto il dubbio, tuttavia l'indagine, come accennato in principio, è stata condotta con una scrupolosità da additare ad esempio a chi si accinga a qualunque lavoro di ricerca.

\section{LuCio BraconI}

VoGEL, F.: Über die Erblichkeit des normalen Elektroenzephalogramms. kart., 92 S., Preis DM - G. Thieme Verlag, Stuttgart, 1958.

Bei der steigenden Bedeutung des Elektroenzephalogramms und seiner Auswertung für die verschiedensten pathologischen Esrscheinungen im Bereich des ZNS wird die Frage der Erblichkeit des normalen Elektroenzephalogramms nicht nur den Humangenetiker, son- dern auch den Kliniker, gleich welcher Fachrichtung, besonders interessieren.

Zur Klärung dieser Frage hat der Verf. 110 erbgleiche (EZ, MZ) und 98 erbverschiedene (DZ, ZZ) Zwillingspaare im Alter von sechs bis zu dreissig Jahren, mit Schwerpunkt der Gruppe der $10-16$ Jährigen, untersucht. Bei jeder untersuchung wurden vom jeweiligen Probanden acht Ableitungen in Ruhe, bei Belastung mit Hyperventilation und $\mathrm{O}_{2}$-Mangel und im natürlichen Schlaf angefertigt. Dabei konnte festgestellt werden, dass im Ruhe-EEG der Grundrhythmus bei EZ (MZ) stets, bei ZZ (DZ) dagegen nur in einem Drittel der Fälle gleich war. Auch bei der Einzelwertberechnung (Alpha-Index, als neues Mass Subalpha- $\%$, duchschnittliche Amplitude, mit einem neu angegebenen Planimeter gemessene Phasenkoordination und weitere qualitative Merkmale) konnte ein deutliches Überwiegen konkordanter Realstionen bei EZ (MZ) gegenüber ZZ (DZ) festgestellt werden. Während im Schlaf-EEG bei einem Merkmalsvergleich mit qualitativen Merkmalen bei EZ (MZ) fast völlige Konkordanz angetroffen wurde, fand sich bei $\mathrm{ZZ}(\mathrm{DZ})$ fast ausschliesslich Diskordanz.

Schliesslich weist der Verf. auf Messfehler seiner neuen Methode und darauf hin, dass auch bei seinen Untersuchungen das Problem nicht nur in der Frage einer grösseren Ähnlichkeit bei EZ (MZ) gegenüber ZZ (DZ), sondern auch in einer Beobachtung der unterschiedlichen Verhältnisse bei EZ (MZ) über längere Zeit hinaus für eine klare genetische Deutung liegt.

Die Arbeit verdient nicht nur in ihrer Fragestellung, sondern auch in der klaren kritischen Darstellung der ausgewerteten Befunde, in der Stellungnahme zu bisher vorliegenden Untersuchungen anderer Autoren und besonders auch in der Deutung und Auswertung der erhobenen Befunde im Rahmen der Zwillingsuntersuchungen der letzten Jahre besondere Beachtung.

H. Grebe, Frankenberg 